\title{
On causality in nonlinear vacuum electrodynamics of the Plebański class
}

\author{
Gerold O. Schellstede • Volker Perlick • \\ Claus Lämmerzahl
}

July 3,2021

Keywords Nonlinear vacuum electrodynamics, Plebański class, Born-Infeld theory, optical metric, light cone structure, causality

Abstract We investigate the Plebański class of electrodynamical theories, i.e., theories of nonlinear vacuum electrodynamics that derive from a Lorentzinvariant Lagrangian (or Hamiltonian). In any such theory the light rays are the lightlike geodesics of two optical metrics that depend on the electromagnetic background field. A set of necessary and sufficient conditions is found whose fulfillment secures that the optical metrics are causal in the sense that the light rays are lightlike or timelike with respect to the underlying space-time metric. Thereupon we derive conditions on the Lagrangian, or the Hamiltonian, of the theory such that the causality conditions are satisfied for all background fields. (The allowed values of the field strength tensor are those for which the excitation tensor is finite and real.) The general results are illustrated with several examples.

\section{Introduction}

In 1912 Gustav Mie made the first attempt to alter Maxwell's theory of vacuum electromagnetism in a way that leads to a consistent theory of point charges 1, 2. Despite the fact that Mie's theory is not gauge-invariant and therefore had to be abandoned, it strongly stimulated the search for nonlinear modifications of Maxwell's vacuum theory [3]. This led in particular to the

G. O. Schellstede, E-mail: gerold.schellstede@zarm.uni-bremen.de, schellst@physik.fu-berlin.de

ZARM, University of Bremen, Am Fallturm, 28359 Bremen, Germany

V. Perlick, E-mail: volker.perlick@zarm.uni-bremen.de

ZARM, University of Bremen, Am Fallturm, 28359 Bremen, Germany

C. Lämmerzahl, E-mail: claus.laemmerzahl@zarm.uni-bremen.de

ZARM, University of Bremen, Am Fallturm, 28359 Bremen, Germany and

Institute of Physics, University of Oldenburg, 26111 Oldenburg, Germany 
nonlinear electrodynamical theory first published by Born in 1933 [4] and then, in a modified form, by Born and Infeld in 1934 [5. Nowadays the Born-Infeld theory experiences a renaissance since Tseytlin [6] pointed out that it can be derived as an effective theory from some string theories.

After the development of quantum electrodynamics (QED) another remarkable attempt was made from a different direction in 1936 by Heisenberg and Euler [7] who derived a nonlinear effective classical theory of vacuum electrodynamics incorporating some effects from QED.

The Born, Born-Infeld and Heisenberg-Euler theories are examples of the so-called Plebański class of nonlinear electrodynamics. This class comprises all theories that can be derived from a Lorentz-invariant Lagrangian (or Hamiltonian). A comprehensive study of this class of theories was brought forward by Plebański [8]; important early contributions were also made by Boillat 9 .

It is a characteristic feature of nonlinear electrodynamical theories that the propagation of light is influenced by an electromagnetic background field. In the geometric optics approximation light propagation can be described in terms of rays. It was shown by Novello et al. 10 that for every theory of the Plebański class the light rays are the lightlike geodesics of two optical metrics which depend on the electromagnetic background field. The two optical metrics are associated with two different polarisation states. This result was rederived, using a different representation, by Obhukov and Rubilar 11 who also showed that the optical metrics are always of Lorentzian signature if non-degenerate.

As a consequence, we have three metrics of Lorentzian signature on the space-time manifold when a theory of the Plebański class and an electromagnetic background field has been specified: The spacetime metric which is assumed to be given from the outset, and the two optical metrics. This raises the question of how the light-cones of these three metrics are related to each other. Important results in this direction have been found already by Goulart and Perez Bergliaffa 12 and in particular by Abalos et al. 13. In this paper we want to further elaborate on this question. In particular, it is our goal to give a necessary and sufficient condition for the optical metrics to be causal. Here and in the following we use the following terminology. We say that the optical metrics are causal if all of their lightlike geodesics are timelike or lightlike with respect to the space-time metric. If we adopt the usual interpretation of (special or general) relativity according to which the light-cone of the spacetime metric determines the maximal speed for signals, causality of the optical metrics is necessary to make sure that light rays do not violate the speed limit for signals. In this sense, nonlinear electrodynamical theories where at least one of the two optical metrics violates the causality condition have to be considered as unphysical.

Without loss of generality, we will assume that the underlying space-time metric is the Minkowski metric, i.e., we will restrict to special relativity. As all the conditions on the light-cone structure are purely algebraic, the results immediatetly carry over to general relativity: We just have to apply them to the light-cones on each tangent space. 
The paper is organised as follows. In section 2 we consider a Lagrangian of the Plebański class and we recall how the characteristic equation (or, equivalently, the dispersion relation or the Fresnel equation) can be written down if a background field has been specified. In sections 3 and 4 we decompose the characteristic equation in terms of the two optical metrics and we derive inequalities that guarantee causality of the optical metrics. These inequalities are further evaluated in section 5 . In section 6 we derive conditions on the Lagrangian that guarantee causality of the optical metrics for all background fields. This question was not touched upon in the above-mentioned articles [12, 13. In section 7 we rewrite these conditions in terms of a Hamiltonian, rather than a Lagrangian, formulation. In section 8 we illustrate our results with some examples.

\section{The characteristic equation}

We consider Minkowski spacetime in standard inertial coodinates, i.e., with the (covariant) components of the metric tensor $\eta_{i k}=\operatorname{diag}(1,1,1,-1)$. We use Einstein's summation convention for Latin indices running from 1 to 4 . Indices are lowered and raised with $\eta_{i k}$ and its inverse $\eta^{i k}$, respectively.

The electromagnetic theories we are interested in are characterised by a Lagrangian $\mathcal{L}(F, G)$. Here

$$
F=\frac{1}{2} F_{m n} F^{m n} \quad \text { and } \quad G=-\frac{1}{4} F_{m n} \tilde{F}^{m n}
$$

are the Lorentz invariants of the electromagnetic field strength tensor $F_{a b}$;

$$
\tilde{F}^{m n}=\frac{1}{2} \varepsilon^{m n a b} F_{a b}
$$

is the Hodge dual of the field strength tensor, with $\varepsilon^{m n a b}$ denoting the totally antisymmetric Levi-Civita tensor, $\varepsilon^{1234}=-1$.

The universal Maxwell equations are

$$
\partial_{[a} F_{b c]}=0
$$

and

$$
\partial_{b} H^{a b}=\frac{4 \pi}{c} j^{a},
$$

where a square bracket around indices means antisymetrisation. The constitutive law that relates the excitation tensor $H^{a b}$ to the field strength tensor $F_{m n}$ is determined by the Lagrangian,

$$
H^{a b}=-\frac{\partial \mathcal{L}}{\partial F_{a b}}=-2 \mathcal{L}_{F} F^{a b}+\mathcal{L}_{G} \tilde{F}^{a b} .
$$

Here and in the following we use the abbreviations

$$
\mathcal{L}_{F}=\frac{\partial \mathcal{L}}{\partial F}, \quad \mathcal{L}_{G}=\frac{\partial \mathcal{L}}{\partial G},
$$




$$
\mathcal{L}_{F F}=\frac{\partial^{2} \mathcal{L}}{\partial F^{2}}, \quad \mathcal{L}_{G G}=\frac{\partial^{2} \mathcal{L}}{\partial G^{2}}, \quad \mathcal{L}_{F G}=\frac{\partial^{2} \mathcal{L}}{\partial F \partial G}
$$

It is the constitutive law (5) that distinguishes different theories, while the Maxwell equations (3) and (4) are always the same. This class of theories, with an arbitrary Lagrangian of the form $\mathcal{L}(F, G)$, is called the Plebanski class. From (5) we read that the standard Maxwell vacuum theory $H^{m n}=F^{m n}$ is included with

$$
\mathcal{L}(F)=-\frac{F}{2} .
$$

In this paper we will restrict to the geometric optics approximation. In this approximation light propagation is determined by a characteristic equation (or eikonal equation) which was derived, for an arbitrary theory of the Plebański class, by Novello et al. [10] and, in a different way, by Obukhov and Rubilar [11. Here we use the same representation as in Schellstede et al. [14. Then the characteristic equation reads

$$
\mathcal{L}_{F}\left\{M \eta^{i j} \eta^{k l}+N \eta^{i j} F^{k m} F_{m}^{l}+P F^{i m} F_{m}^{j} F^{k n} F_{n}^{l}\right\} p_{i} p_{j} p_{k} p_{l}=0
$$

with

$$
\begin{gathered}
M:=\mathcal{L}_{F}^{2}+2 \mathcal{L}_{F} \mathcal{L}_{F G} G-\frac{1}{2} \mathcal{L}_{F} \mathcal{L}_{G G} F+\left(\mathcal{L}_{F G}^{2}-\mathcal{L}_{F F} \mathcal{L}_{G G}\right) G^{2}, \\
N:=2 \mathcal{L}_{F} \mathcal{L}_{F F}+\frac{1}{2} \mathcal{L}_{F} \mathcal{L}_{G G}+\left(\mathcal{L}_{F G}^{2}-\mathcal{L}_{F F} \mathcal{L}_{G G}\right) F \\
P:=\mathcal{L}_{F F} \mathcal{L}_{G G}-\mathcal{L}_{F G}^{2} .
\end{gathered}
$$

The characteristic equation is to be viewed as a partial differential equation for a function $\psi\left(x^{i}\right)$. If $p_{i}=\partial \psi / \partial x^{i}$ is a nowhere vanishing solution of the eikonal equation, the function $\psi$ foliates the space-time manifold into hypersurfaces $\psi=$ constant which are called characteristic surfaces or wave surfaces. If read as an algebraic equation for the covector $p_{i},(9)$ is called the dispersion relation or the Fresnel equation.

\section{Causality of the optical metrics in the case $M \neq 0$}

In the case $M \neq 0$ the characteristic equation is decomposable into a product of the form

$$
\mathcal{L}_{F} Q_{+} Q_{-}=0
$$

where

$$
Q_{ \pm}=a_{ \pm}^{i k} p_{i} p_{k}
$$

with

$$
\begin{gathered}
a_{ \pm}^{i k}=\eta^{i k}+\sigma_{ \pm} F^{i l} F_{l}^{k} \\
\sigma_{ \pm}=\frac{N}{2 M} \pm \sqrt{\frac{N^{2}}{4 M^{2}}-\frac{P}{M}}
\end{gathered}
$$


$a_{+}^{i k}$ and $a_{-}^{i k}$ are known as the optical metrics. As

$$
N^{2}-4 M P=\left(2 \mathcal{L}_{F} \mathcal{L}_{F F}-\frac{1}{2} \mathcal{L}_{F} \mathcal{L}_{G G}-P F\right)^{2}+4\left(\mathcal{L}_{F} \mathcal{L}_{F G}-P G\right)^{2}
$$

is a sum of two squares, the optical metrics are always real. Note that we are free to multiply each of the optical metrics with a non-zero factor, which may depend on the foot-point, without changing the characteristic equation.

If

$$
\operatorname{Det}\left|a_{ \pm}^{i k}\right|=-\left(1+\sigma_{ \pm} F-\sigma_{ \pm}^{2} G^{2}\right)^{2}
$$

does not vanish, we may introduce the contravariant components $b_{i k}^{ \pm}$of the optical metrics via

$$
a_{ \pm}^{i k} b_{k l}^{ \pm}=\delta_{l}^{i}
$$

Here we have to keep in mind that we agreed to raise and to lower indices with the space-time metric, so in general $b_{i k}^{ \pm} \neq a_{ \pm i k} \equiv \eta_{i m} \eta_{k n} a_{ \pm}^{m n}$. With the help of the well-known identities (see Plebanski [8])

$$
\tilde{F}_{m n} F^{n k}=G \delta_{m}^{k}, \quad-F_{m n} F^{n k}+\tilde{F}_{m n} \tilde{F}^{n k}=F \delta_{m}^{k}
$$

it is easy to verify that (15) can be rewritten as

$$
a_{ \pm}^{i k}=\left(1+\sigma_{ \pm} F\right) \eta^{i k}+\sigma_{ \pm} \tilde{F}^{i l} \tilde{F}_{l}^{k}
$$

and that

$$
b_{i k}^{ \pm}=\frac{\left(1+\sigma_{ \pm} F\right) \eta_{i k}-\sigma_{ \pm} F_{i}{ }^{l} F_{k l}}{1+\sigma_{ \pm} F-\sigma_{ \pm}^{2} G^{2}}=\frac{\eta_{i k}-\sigma_{ \pm} \tilde{F}_{i}^{l} \tilde{F}_{k l}}{1+\sigma_{ \pm} F-\sigma_{ \pm}^{2} G^{2}} .
$$

From (18) we read that the determinant of the optical metric cannot be positive. As a consequence the signature must be Lorentzian, i.e. $(+++-)$ or $(---+)$, if the determinant is non-zero. This result was already found by Obukhov and Rubilar [11.

We first observe that we have to require $\mathcal{L}_{F} \neq 0$ because otherwise any $p_{i}=\partial \psi / \partial x^{i}$ satisfies the characteristic equation which cannot be considered to be a reasonable law of light propagation. Then the characteristic equation (9) splits into two equations

$$
a_{ \pm}^{i k} \frac{\partial \psi}{\partial x^{i}} \frac{\partial \psi}{\partial x^{k}}=0
$$

with the optical metrics given by (15). Each of these two equations has the form of a Hamilton-Jacobi equation with the Hamiltonian $Q_{ \pm}=a_{ \pm}^{i k} p_{i} p_{k}$. The corresponding set of Hamilton equations

$$
\begin{gathered}
\dot{x}^{i}=\frac{\partial Q_{ \pm}}{\partial p_{i}}=2 a_{ \pm}^{i k} p_{k}=2 \eta^{i k} p_{k}-2 \sigma_{ \pm} F^{i l} F_{l}{ }^{k} p_{k} \\
\dot{p}_{i}=-\frac{\partial Q_{ \pm}}{\partial x^{i}}=-p_{m} p_{n} \frac{\partial a_{ \pm}^{m n}}{\partial x^{i}} \\
Q_{ \pm}=a_{ \pm}^{i k} p_{i} p_{k}=0
\end{gathered}
$$


determines the bicharacteristic curves or rays. For background material on the notion of bicharacteristic curves we refer to Courant and Hilbert [15.

If the determinant (18) is non-zero, the covariant components (22) of the optical metrics are well defined and the Levi-Civita derivative $\nabla^{ \pm}$of the optical metric $b_{i k}^{ \pm}$exists. Then the Hamilton equations (24), (25) and (26) require that the rays are null geodesics of the optical metric,

$$
\dot{x}^{k} \nabla_{k}^{ \pm} \dot{x}^{i}=0, \quad p_{i}=\frac{1}{2} b_{i k}^{ \pm} \dot{x}^{k}, \quad b_{i k}^{ \pm} \dot{x}^{i} \dot{x}^{k}=0 .
$$

For the rest of this section we fix one of the two optical metrics, $a_{A}^{i k}$, where the index $A$ stands for + or for - . We want to characterise the case that the optical metric $a_{A}^{i k}$ is non-degenerate and causal in the sense that, for any solution to (24) with (26), the vector $\dot{x}^{i}$ satisfies $\eta_{i k} \dot{x}^{i} \dot{x}^{k} \leq 0$. The non-degeneracy condition assures that there is a sphere's worth of directions tangent to rays at each point, i.e., that it is possible to send a ray in each spatial direction, and the causality condition assures that the rays are timelike or lightlike with respect to the space-time metric. The latter condition has to be fulfilled if we assume that the maximal speed for signals is determined by the null cone of the space-time metric.

We know already that the non-degeneracy condition is satisfied if and only if $1+\sigma_{A} F-\sigma_{A}^{2} G^{2} \neq 0$, so we only have to investigate the causality condition. If $\sigma_{A}=0$, the optical metric coincides with the space-time metric and the causality condition is obviously satisfied, so it only remains to investigate the case $\sigma_{A} \neq 0$. Moeover, if a covector $p_{i}$ is a principal null covector of the electromagnetic field, i.e., if $F^{j i} p_{i}$ is a multiple of $\eta^{j i} p_{i}$, the corresponding ray velocity $\dot{x}^{i}=2 a_{A}^{i k} p_{k}$ is lightlike with respect to the space-time metric, so for these covectors the causality condition is never violated. Therefore, what we have to investigate is the case that $1+\sigma_{A} F-\sigma_{A}^{2} G^{2} \neq 0$ and $\sigma_{A} \neq 0$, and we have to find a condition such that, for all solutions $p_{i}$ of (26) that are not principal null covectors, the corresponding vector $\dot{x}^{i}=2 a_{A}^{i k} p_{k}$ satisfies $\eta_{i j} \dot{x}^{i} \dot{x}^{j} \leq 0$.

To work this out, we fix such a covector $p_{i}$. Then the four vectors

$$
p^{k}=\eta^{k l} p_{l}, \quad \dot{x}^{k}=2 a_{A}^{k l} p_{l}, \quad \xi^{k}=F^{k l} p_{l}, \quad \Xi^{k}=\tilde{F}^{k l} p_{l}-\sigma_{A} G F^{k l} p_{l}
$$

are linearly independent, which follows from the fact that $p_{i}$ is assumed not to be a principal null covector. With the help of the identities (20) one verifies that

$$
p_{k} \xi^{k}=p_{k} \Xi^{k}=\dot{x}_{k} p^{k}=\dot{x}_{k} \xi^{k}=\dot{x}_{k} \Xi^{k}=\xi^{k} \Xi_{k}=0
$$

and

$$
\begin{aligned}
& p^{i} p_{i}=-\sigma_{A} \xi^{i} \xi_{i} ; \quad\left(1+\sigma_{A} F-\sigma_{A}^{2} G^{2}\right) p^{i} p_{i}=-\sigma_{A} \Xi^{i} \Xi_{i} ; \\
& \frac{\dot{x}_{k} \dot{x}^{k}}{4}=-\left(1+\sigma_{A} F-\sigma_{A}^{2} G^{2}\right) p^{i} p_{i} .
\end{aligned}
$$

These relations fix the causal character of each of the four basis vectors in dependence of the signs of $1+\sigma_{A} F-\sigma_{A}^{2} G^{2}$ and $\sigma_{A}$ : 
1) $1+\sigma_{A} F-\sigma_{A}^{2} G^{2}<0 ; \quad \sigma_{A}>0$

$$
\Rightarrow \quad p^{i} p_{i}>0 ; \quad \dot{x}_{k} \dot{x}^{k}>0 ; \quad \xi^{i} \xi_{i}<0 ; \quad \Xi^{i} \Xi_{i}>0
$$

2) $1+\sigma_{A} F-\sigma_{A}^{2} G^{2}<0 ; \quad \sigma_{A}<0$

$$
\Rightarrow \quad p^{i} p_{i}>0 ; \quad \dot{x}_{k} \dot{x}^{k}>0 ; \quad \xi^{i} \xi_{i}>0 ; \quad \Xi^{i} \Xi_{i}<0
$$

3) $1+\sigma_{A} F-\sigma_{A}^{2} G^{2}>0 ; \quad \sigma_{A}>0$

$$
\Rightarrow \quad p^{i} p_{i}<0 ; \quad \dot{x}_{k} \dot{x}^{k}>0 ; \quad \xi^{i} \xi_{i}>0 ; \quad \Xi^{i} \Xi_{i}>0
$$

4) $1+\sigma_{A} F-\sigma_{A}^{2} G^{2}>0 ; \quad \sigma_{A}<0$

$$
\Rightarrow \quad p^{i} p_{i}>0 ; \quad \dot{x}_{k} \dot{x}^{k}<0 ; \quad \xi^{i} \xi_{i}>0 ; \quad \Xi^{i} \Xi_{i}>0
$$

We see that the causality condition is satisfied only in Case 4). In combination with our earlier observation that the causality condition is always satisfied if $\sigma_{A}=0$ we can summarise the result of this section in the following way:

In the case $M \neq 0$ with $\mathcal{L}_{F} \neq 0$ the optical metric $a_{A}^{i k}$ is non-degegenerate and causal if and only if

$$
1+\sigma_{A} F-\sigma_{A}^{2} G^{2}>0 ; \quad \sigma_{A} \leqq 0 .
$$

Note that for deriving this result we have not used that $\sigma_{A}$ is given by the specific expression (16), hence it is true for a metric of the form (15) with any $\sigma_{A}$.

Moreover, we mention that the causality condition (31) can also be derived with the help of the results of Abalos et al. [13. However, the derivation given here is simpler because it was not necessary to analyse the eigenvalue problem of the optical metric.

\section{Causality of the optical metrics in the case $M=0$}

In the case $M=0$ the characteristic equation (9) again factorises,

$$
\mathcal{L}_{F} Q_{1} Q_{2}=0
$$

where

$$
Q_{1 / 2}=a_{1 / 2}^{i k} p_{i} p_{k},
$$

but this time the optical metrics are

$$
a_{1}^{i j}=N \eta^{i j}+P F^{i m} F_{m}^{j}, \quad a_{2}^{i j}=F^{i m} F_{m}^{j} .
$$

As in the case $M \neq 0$ we have to require $\mathcal{L}_{F} \neq 0$ because otherwise the characteristic equation is satisfied identically. In analogy to the procedure in the previous section we will investigate under what conditions the optical metrics are non-degenerate and causal. 
In the following we make use of the fact that each optical metric is determined uniquely only up to a non-zero factor which may depend on the foot-point. If $N \neq 0$, we can divide the optical metric $a_{1}^{i j}$ by $N$. Then the results of the previous section apply, with $\sigma_{A}$ replaced by $P / N$. If $N=0$ and $P \neq 0$, we can divide $a_{1}^{i j}$ by $\mathrm{P}$. Then the two optical metrics coincide, so we only have to discuss $a_{2}^{i j}$ which we will do immediately. The case $N=P=0$ is obviously forbidden by the non-degeneracy condition.

For the determinant of the metric $a_{2}^{i k}$ one gets

$$
\operatorname{Det}\left|a_{2}^{i k}\right|=-G^{4}
$$

so the non-degeneracy condition requires $G \neq 0$. For the Hamiltonian $Q_{2}=$ $a_{2}^{i k} p_{i} p_{k}$ Hamilton's equations read

$$
\begin{gathered}
\dot{x}^{i}=\frac{\partial Q_{2}}{\partial p_{i}}=2 a_{2}^{i k} p_{k}=2 F^{i m} F_{m}^{k} p_{k}, \\
\dot{p}_{i}=\frac{\partial Q_{2}}{\partial x^{i}}=p_{j} p_{k} \frac{\partial a_{2}^{j k}}{\partial x^{i}}=p_{j} p_{k} \frac{\partial\left(F^{j m} F^{k}{ }_{m}\right)}{\partial x^{i}}, \\
Q_{2}=a_{2}^{i k} p_{i} p_{k}=F^{i m} F^{k}{ }_{m} p_{i} p_{k}=0 .
\end{gathered}
$$

We will now prove that the causality condition $\eta_{i k} \dot{x}^{i} \dot{x}^{k} \leq 0$ cannot hold for all solutions of these equations if the non-degeneracy condition $G \neq 0$ is satisfied.

To that end we choose a non-zero covector $p_{i}$ that satisfies (38) and is not a principal null covector of the electromagnetic field, i.e., $\xi^{k}:=F^{k l} p_{l}$ and $p^{k}$ are linearly independent. Such a covector exists because it is well known that for an electromagnetic field with $G \neq 0$ there are precisely two linearly independent principal null covectors, see e.g. the Appendix of Abalos et al. [13. Then we find from (38) and from the antisymmetry of $F^{k l}$ that

$$
\xi_{k} \xi^{k}=0, \quad \xi^{k} p_{k}=0
$$

As $\xi^{k}$ and $p^{k}$ are linearly independent, these two conditions imply that $p^{k}$ is spacelike with respect to the space-time metric, $p_{k} p^{k}>0$. On the other hand, we find from (36) that

$$
\dot{x}^{k} \dot{x}_{k}=4 G^{2} p^{i} p_{i},
$$

so the non-degeneracy condition $G \neq 0$ yields $\dot{x}^{k} \dot{x}_{k}>0$, i.e., the causality condition is necessarily violated.

We summarise the result of this section in the following way: In the case $M=0$ with $\mathcal{L}_{F} \neq 0$ it is impossible that both optical metrics are nondegenerate and causal.

Note that the results of this section are irrelevant for the case of $\mathcal{L}(F)$ theories, i.e., for Lagrangians that are independent of $G$, because in this case the condition $M \neq 0$ is equivalent to $\mathcal{L}_{F} \neq 0$. 


\section{Evaluation of the causality conditions for both optical metrics}

In this short section we evaluate the causality condition (31) for the case that it holds both for $\sigma_{+}$and for $\sigma_{-}$. In view of $\sigma_{ \pm}$given by (16) we assume that $\sigma_{+} \geqq \sigma_{-}$but otherwise the specific form of $\sigma_{ \pm}$is not used in this section. We assume $G \neq 0$ and treat the case $G=0$ in the end as a limit.

For any given $F$ and $G \neq 0$, we define a real-valued function $T$ by

$$
T(x):=x^{2} G^{2}-x F-1
$$

such that the condition $1+\sigma_{A} F-\sigma_{A}^{2} G^{2}>0$ is equivalent to $T\left(\sigma_{A}\right)<0$ Obviously, the roots of $T(x)=0$ are

$$
x_{ \pm}=\frac{F}{2 G^{2}} \pm \sqrt{\frac{F^{2}}{4 G^{4}}+\frac{1}{G^{2}}}
$$

with $x_{+}>0$ and $x_{-}<0$. Moreover, the only extremum (minimum) of $T(x)$ is given by

$$
T^{\prime}\left(x_{m}\right)=2 x_{m} G^{2}-F=0 \quad \Rightarrow \quad x_{m}=\frac{F}{2 G^{2}} \quad \text { with } \quad T^{\prime \prime}\left(x_{m}\right)=2 G^{2} .
$$

Therefore $T(x)<0$ together with $x \leqq 0$ is equivalent to

$$
0 \geqq x>\frac{F}{2 G^{2}}-\sqrt{\frac{F^{2}}{4 G^{4}}+\frac{1}{G^{2}}} .
$$

This is satisfied both by $x=\sigma_{+}$and by $x=\sigma_{-}$if and only if

$$
0 \geqq \sigma_{+}, \quad \sigma_{-}>\frac{F}{2 G^{2}}-\frac{1}{G^{2}} \sqrt{\frac{F^{2}}{4}+G^{2}} .
$$

These conditions are also true in the case $G=0$. For $G \rightarrow 0$, the second inequality is automatically satisfied $\left(\sigma_{-}>-\infty\right)$ if $F \leq 0$ and it requires $\sigma_{-}>-1 / F$ if $F>0$. This can be easily seen by Taylor expanding the squareroot about $G=0$.

\section{Causality conditions on the Lagrangian}

The results of the preceding sections allow us to check if causality holds if not only the the Lagrangian but also the background field has been specified. In this section we want to derive conditions on the Lagrangian that guarantee causality for all allowed background fields. Note that, in general, not all values of $F$ and $G$ are allowed. The best known counter-example is the Born-Infeld theory, see section 8.2 below, where the Lagrangian involves a square-root and only those values of $F$ and $G$ are allowed for which the expression under the square-root is positive. In this section we will not specify the allowed values of $F$ and $G$ but we will assume that they form a connected domain in the $(F, G)$-plane that contains the point $(F, G)=(0,0)$. 
We have seen that a reasonable law of light propagation requires $\mathcal{L}_{F} \neq 0$. If this condition is supposed to hold for all allowed values of $F$ and $G$, continuity implies that either $\mathcal{L}_{F}<0$ or $\mathcal{L}_{F}>0$ on the entire domain of allowed values. For the following discussion we assume that

$$
\mathcal{L}_{F}<0
$$

because this includes the standard Maxwell vacuum case which, with our conventions, is given by (8). We will then get the corresponding results for the case $\mathcal{L}_{F}>0$ easily at the end of the section by replacing $\mathcal{L}$ with $-\mathcal{L}$. Note that in principle we are free to multiply the Lagrangian with a non-zero constant factor, positive or negative, without changing the characteristic equation. We just have to keep in mind that then we also have to multiply the right-hand side of the constitutive law (5) with the same factor.

To investigate the causality conditions on the Lagrangian we first recall from the preceding sections that causality of both optical metrics is possible only if $M \neq 0$. As $M=\mathcal{L}_{F}^{2}>0$ for $(F, G)=(0,0)$, continuity requires

$$
M>0
$$

on the entire domain of allowed values.

In combination with (46) and (47) we have to evaluate the two inequalities (45). With $\sigma_{+}$given by (16), the first inequality requires

$$
\frac{N}{2 M}+\sqrt{\frac{N^{2}}{4 M^{2}}-\frac{P}{M}} \leq 0 .
$$

As $M>0$, this is true if and only if

$$
N \leq 0, \quad P \geq 0 .
$$

The second inequality in (45) requires

$$
\frac{N}{2 M}-\sqrt{\frac{N^{2}}{4 M 2}-\frac{P}{M}}>\frac{F}{2 G^{2}}-\sqrt{\frac{F^{2}}{4 G^{4}}+\frac{1}{G^{2}}} .
$$

We evaluate this condition first for the case that $P=0$ and then for the case that $P \neq 0$. If $P=0$, (50) simplifies to

$$
\frac{N}{M}>\frac{F}{2 G^{2}}-\sqrt{\frac{F^{2}}{4 G^{4}}+\frac{1}{G^{2}}}
$$

where we have used that $N \leq 0$. Multiplication with the strictly positive factor $\left(F+\sqrt{F^{2}+4 G^{2}}\right) /\left(2 G^{2}\right)$ results in

$$
M>-\frac{N}{2}\left(F+\sqrt{F^{2}+4 G^{2}}\right) .
$$

Note that (52) implies $M>0$, so the latter condition need not be stated separately. 
In the case $P \neq 0$, (49) requires $P>0$. Multiplication of (50) with the strictly positive factor $\left(-N-\sqrt{N^{2}-4 M P}\right)\left(F+\sqrt{F^{2}+4 G^{2}}\right) /\left(4 M G^{2}\right)$ results in

$$
-N-P\left(F+\sqrt{F^{2}+4 G^{2}}\right)>\sqrt{N^{2}-4 M P} .
$$

This inequality is equivalent to the conditions that the left-hand side is positive and that the inequality is true with both sides squared. These two conditions read

$$
\begin{gathered}
N<-P\left(F+\sqrt{F^{2}+4 G^{2}}\right), \\
M>-P G^{2}-\frac{1}{2}(N+P F)\left(F+\sqrt{F^{2}+4 G^{2}}\right) .
\end{gathered}
$$

(54) implies $N<0$, and both conditions together imply $M>0$. We can summarise the results in the following way. For a Lagrangian with $\mathcal{L}_{F}<0$ causality holds if and only if for all allowed background fields

$$
\begin{gathered}
P \geq 0, \\
N \leq-P\left(F+\sqrt{F^{2}+4 G^{2}}\right), \\
M>-P G^{2}-\frac{1}{2}(N+P F)\left(F+\sqrt{F^{2}+4 G^{2}}\right),
\end{gathered}
$$

where the equality sign in (57) can hold only if $P=0$.

Inserting the definitions of $M, N$ and $P$ into these expressions results in

$$
\begin{gathered}
\mathcal{L}_{F F} \mathcal{L}_{G G} \geq \mathcal{L}_{F G}^{2} \\
2 \mathcal{L}_{F} \mathcal{L}_{F F}+\frac{1}{2} \mathcal{L}_{F} \mathcal{L}_{G G} \leq-\left(\mathcal{L}_{F F} \mathcal{L}_{G G}-\mathcal{L}_{F G}^{2}\right) \sqrt{F^{2}+4 G^{2}} \\
\mathcal{L}_{F}<-F \mathcal{L}_{F F}+\frac{1}{4} F \mathcal{L}_{G G}-2 G \mathcal{L}_{\mathrm{FG}} \\
-\left(\mathcal{L}_{F F}+\frac{1}{4} \mathcal{L}_{G G}\right) \sqrt{F^{2}+4 G^{2}}=: \alpha
\end{gathered}
$$

where equality in (60) can hold only if $\mathcal{L}_{F F} \mathcal{L}_{G G}=\mathcal{L}_{F G}^{2}$. For theories with $\mathcal{L}_{F}<0$, the conditions (59), (60) and (61) are equivalent to

$$
\begin{gathered}
\mathcal{L}_{F F} \mathcal{L}_{G G} \geq \mathcal{L}_{F G}^{2}, \\
\mathcal{L}_{F F} \geq 0, \quad \mathcal{L}_{G G} \geq 0, \\
\mathcal{L}_{F}<-F \mathcal{L}_{F F}+\frac{1}{4} F \mathcal{L}_{G G}-2 G \mathcal{L}_{\mathrm{FG}}-\left(\mathcal{L}_{F F}+\frac{1}{4} \mathcal{L}_{G G}\right) \sqrt{F^{2}+4 G^{2}} .
\end{gathered}
$$

To prove this, it is easy to see that (59) and (60) imply (63). Conversely, one can verify with a bit of algebra that (62) and (63) imply $\alpha \leq 0$ where $\alpha$ is defined in (61); with this information at hand, it can be shown that (62), (63) and (64) imply (60).

We have derived the causality conditions here for Lagrangians with $\mathcal{L}_{F}<0$. The results for the case $\mathcal{L}_{F}>0$ follow immediately by replacing $\mathcal{L}$ with $-\mathcal{L}$. 
Then (62) remains unchanged whereas the inequality signs in (63) and (64) have to be reversed.

For an $\mathcal{L}(F)$-theory, i.e., if the Lagrangian is independent of $G$, the causality conditions (62), (63) and (64)drastically simplify to

$$
\begin{gathered}
\mathcal{L}_{F F} \geq 0, \\
-\mathcal{L}_{F}-\mathcal{L}_{F F} F>\mathcal{L}_{F F} \sqrt{F^{2}+4 G^{2}} .
\end{gathered}
$$

The striking feature of (66) is that the left-hand side is independent of $G$. If $\mathcal{L}_{F F} \neq 0$, the right-hand side increases with $G$, so for any allowed $F$ we can find a sufficiently big value of $G$ such that (66) is violated. Here it is crucial that all values of $G$ are allowed because a Lagrangian of the form $\mathcal{L}(F)$ and therefore the resulting field equations can restrict only the values of $F$ but not those of $G$. In other words, any $\mathcal{L}(F)$-theory violates the causality condition for some allowed field values unless $\mathcal{L}_{F F}$ is identically zero. The latter case is just the standard Maxwell vacuum theory.

\section{Causality conditions in the Hamiltonian formulation}

As an alternative to the Lagrangian formalism we have used so far, one can introduce a Hamiltonian formalism. The basic equations are well-known since the pioneering work of Plebański 8 . The passage from the Lagrangian to the Hamiltonian formulation is possible if the constitutive law (5) can be solved for the field strength tensor $F_{a b}$.

The Hamiltonian is then derived from the Lagrangian via a Legendre transformation

$$
\mathcal{H}\left(H^{a b}\right)=-\frac{1}{2} H^{m n} F_{m n}-\mathcal{L}\left(F_{a b}\right)
$$

where on the right-hand side the field strength tensor has to be expressed in terms of the excitation. For a theory of the Plebański class the Hamiltonian is a function of the two excitation invariants

$$
R=-\frac{1}{2} H^{a b} H_{a b} \quad \text { and } \quad S=\frac{1}{4} H_{a b} \tilde{H}^{a b} .
$$

In the Hamiltonian formalism the constitutive law reads

$$
F_{a b}=-\frac{\partial \mathcal{H}}{\partial H^{a b}}=2 \mathcal{H}_{R} H_{a b}-\mathcal{H}_{S} \tilde{H}_{a b} .
$$

We have used already in 14 the fact that all equations of the Hamiltonian formalism can be obtained from the corresponding equations in the Lagrangian formalism by the replacement rules

$$
F^{m n} \hookrightarrow \tilde{H}^{m n}, \quad \mathcal{L} \hookrightarrow \mathcal{H} .
$$

As $F^{m n} \hookrightarrow \tilde{H}^{m n}$ implies

$$
\tilde{F}^{m n} \hookrightarrow-H^{m n}, \quad F \hookrightarrow R, \quad G \hookrightarrow S,
$$


the characteristic equation can be rewritten in terms of the Hamiltonian as

$$
\mathcal{H}_{R}\left\{\hat{M} \eta^{i j} \eta^{k l}+\hat{N} \eta^{i j} \tilde{H}^{k m} \tilde{H}_{m}^{l}+\hat{P} \tilde{H}^{i m} \tilde{H}_{m}^{j} \tilde{H}^{k n} \tilde{H}_{n}^{l}\right\} p_{i} p_{j} p_{k} p_{l}=0
$$

with

$$
\begin{aligned}
& \hat{M}=\mathcal{H}_{R}^{2}+2 \mathcal{H}_{R} \mathcal{H}_{R S} S-\frac{1}{2} \mathcal{H}_{R} \mathcal{H}_{S S} R+\left(\mathcal{H}_{R S}^{2}-\mathcal{H}_{R R} \mathcal{H}_{S S}\right) S^{2}, \\
& \hat{N}=2 \mathcal{H}_{R} \mathcal{H}_{R R}+\frac{1}{2} \mathcal{H}_{R} \mathcal{H}_{S S}+\left(\mathcal{H}_{R S}^{2}-\mathcal{H}_{R R} \mathcal{H}_{S S}\right) R, \\
& \hat{P}=\mathcal{H}_{R R} \mathcal{H}_{S S}-\mathcal{H}_{R S}^{2} .
\end{aligned}
$$

In analogy to the discussion in section [2] we have to require $\mathcal{H}_{R} \neq 0$ for a reasonable law of light propagation. With the same argument as in section 4 we find that in the case $\hat{M}=0$ at least one of the optical metrics necessarily violates the causality condition. In the case $\hat{M} \neq 0$, the optical metrics (15) can be rewritten in terms of the Hamiltonian as

$$
a_{ \pm}^{i k}=\eta^{i k}+\hat{\sigma}_{ \pm} \tilde{H}^{i m} \tilde{H}_{m}^{k} \quad \text { with } \quad \hat{\sigma}_{ \pm}=\frac{\hat{N}}{2 \hat{M}} \pm \sqrt{\frac{\hat{N}^{2}}{4 \hat{M}^{2}}-\frac{\hat{P}}{\hat{M}}}
$$

Thereupon, the causality conditions (31) for a background field with $\mathcal{H}_{R} \neq 0$ and $\hat{M} \neq 0$ require

$$
\hat{\sigma}_{A} \leq 0, \quad 1+\hat{\sigma}_{A} R-\hat{\sigma}_{A}^{2} S^{2}>0 .
$$

As for the standard Maxwell vacuum theory $\left(F_{a b}=H_{a b}\right)$ we have $\mathcal{H}_{R}=$ $1 / 2$, according to (69), we write down the analogues of the causality conditions (62), (63) and (64) for the case that $\mathcal{H}_{R}>0$. Applying the replacement rules yields

$$
\begin{gathered}
\mathcal{H}_{R R} \mathcal{H}_{S S}-\mathcal{H}_{R S}^{2} \geq 0, \\
\mathcal{H}_{R R} \leq 0, \quad \mathcal{H}_{S S} \leq 0, \\
\mathcal{H}_{R}>-R \mathcal{H}_{R R}+\frac{R}{4} \mathcal{H}_{S S}-2 S \mathcal{H}_{R S}-\left(\mathcal{H}_{R R}+\frac{1}{4} \mathcal{H}_{S S}\right) \sqrt{R^{2}+4 S^{2}},
\end{gathered}
$$

In analogy to the Lagrangian case, the causality conditions for theories with $\mathcal{H}_{R}<0$ follow from the ones for theories with $\mathcal{H}_{R}>0$ by replacing $\mathcal{H}$ with $-\mathcal{H}$ everywhere.

If the Lagrangian is independent of $G$, the Hamiltonian is independent of $S$, i.e., $\mathcal{L}(F)$-theories correspond to $\mathcal{H}(R)$-theories. For an $\mathcal{H}(R)$-theory with $\mathcal{H}_{R}>0$ the causality conditions simplify to

$$
\begin{gathered}
\mathcal{H}_{R R} \leq 0 \\
\mathcal{H}_{R}+R \mathcal{H}_{R R}>-\mathcal{H}_{R R} \sqrt{R^{2}+4 S^{2}},
\end{gathered}
$$

In analogy to the Lagrangian case we see that only in the Maxwell case $\mathcal{H}_{R R}=$ 0 can these conditions hold for arbitrarily large $S$. 
Here we have chosen the Lagrangian formulation as the starting point and derived the corresponding Hamiltonian formulation under the assumption that the constitutive law (5) can be solved for the field strength. Conversely, one could start out from a Hamiltonian formulation and then perform the passage to the Lagrangian formulation provided that the constitutive law (69) can be solved for the excitation. We emphasize that there are some examples of interest where the theory is formulated in terms of a Hamiltonian and where the constitutive law (69) does not uniquely determine the excitation in terms of the field strength. In those cases the causality conditions (76) to (78) are valid all the same because they can be derived directly from the field equations in terms of the Hamiltonian. An example of this kind will be treated in section 8.6 below.

\section{Examples}

In this section we briefly investigate the causality conditions for some specific theories of the Plebański class.

8.1 Standard Maxwell vacuum theory

The standard Maxwell vacuum theory is an $L(F)$-theory with the Lagrangian

$$
\mathcal{L}=-\frac{F}{2}
$$

hence

$$
\mathcal{L}_{F}=-1 / 2 ; \quad \mathcal{L}_{F F}=0
$$

All values of $F$ and $G$ are allowed and the constitutive law reads

$$
H^{m n}=F^{m n} .
$$

From (82) we read that we have indeed $\mathcal{L}_{F}<0$ and that the causality conditions (65) and (66) are satisfied in a trivial way for all $F$ and $G$. As in the standard Maxwell vacuum theory the optical metrics coincide with the spacetime metric, it is clear from the outset that the causality conditions have to hold.

8.2 Born-Infeld theory

The Born-Infeld theory [5] derives from the Lagrangian

$$
\mathcal{L}(F, G)=-b_{0}^{2} \sqrt{1+\frac{F}{b_{0}^{2}}-\frac{G^{2}}{b_{0}^{4}}}+b_{0}^{2}
$$


where $b_{0}$ is a hypothetical constant of Nature with the dimension of a field strength. For $b_{0} \rightarrow \infty$ the Born-Infeld theory approaches the standard Maxwell vacuum theory.

From the Lagrangian we find

$$
\begin{gathered}
\mathcal{L}_{F}=\frac{-1}{2 \sqrt{1+\frac{F}{b_{0}^{2}}-\frac{G^{2}}{b_{0}^{4}}}}, \\
\mathcal{L}_{G}=\frac{G}{b_{0}^{2} \sqrt{1+\frac{F}{b_{0}^{2}}-\frac{G^{2}}{b_{0}^{4}}}},
\end{gathered}
$$

so the constitutive law (5) reads

$$
H^{a b}=\frac{F^{a b}+\frac{G}{b_{0}^{2}} \tilde{F}^{a b}}{\sqrt{1+\frac{F}{b_{0}^{2}}-\frac{G^{2}}{b_{0}^{4}}}} .
$$

As the excitation has to be real and finite, the allowed values of $F$ and $G$ are restricted by

$$
1+\frac{F}{b_{0}^{2}}-\frac{G^{2}}{b_{0}^{4}}>0
$$

which requires, in particular,

$$
1+\frac{F}{b_{0}^{2}}>0
$$

To demonstrate causality we could check if the causality conditions (62), (63) and (64) are satisfied. However, in this case we prefer to directly verify that the conditions (45) together with $\mathcal{L}_{F}<0$ and $M>0$ are satisfied for all allowed background fields. We read from (85) that $\mathcal{L}_{F}<0$ is obviously true for all allowed field values. By calculating the second derivatives of the Lagrangian we find that

$$
M=\frac{\left(1+\frac{F}{b_{0}^{2}}\right)^{2}}{4\left(1+\frac{F}{b_{0}^{2}}-\frac{G^{2}}{b_{0}^{4}}\right)^{2}},
$$

so the condition $M>0$ is satisfied for all allowed $F$ and $G$. To verify the inequalities (45) we note that the two optical metrics (15) coincide and that they satisfy

$$
\sigma_{+}=\sigma_{-}=\frac{-1}{b_{0}^{2}+F}
$$


and

$$
1+\sigma_{ \pm} F-\sigma_{ \pm}^{2} G^{2}=\frac{1+\frac{F}{b_{0}^{2}}-\frac{G^{2}}{b_{0}^{4}}}{\left(1+\frac{F}{b_{0}^{2}}\right)^{2}}
$$

This demonstrates that the causality conditions (45) are satisfied precisely for the allowed field values.

\subsection{Born theory}

Before introducing the Born-Infeld theory, Born [4] had suggested the Lagrangian

$$
\mathcal{L}(F)=-b_{0}^{2} \sqrt{1+\frac{F}{b_{0}^{2}}}+b_{0}^{2} .
$$

This is an $\mathcal{L}(F)$-theory with

$$
\begin{gathered}
\mathcal{L}_{F}=\frac{-1}{2 \sqrt{1+\frac{F}{b_{0}^{2}}}} \\
\mathcal{L}_{F F}=\frac{1}{4 b_{0}^{2}{\sqrt{1+\frac{F}{b_{0}^{2}}}}^{3}},
\end{gathered}
$$

which leads to the constitutive law

$$
H^{a b}=\frac{F^{a b}}{\sqrt{1+\frac{F}{b_{0}^{2}}}} .
$$

The allowed values of $F$ are restricted by

$$
1+\frac{F}{b_{0}^{2}}>0
$$

while there is no restriction on the values of $G$.

From (94) and (95) we read that $\mathcal{L}_{F}<0$ and that the first causality condition (65) is satisfied for all allowed field values. However, the second causality condition (66) is violated if $G^{2} \geq b_{0}^{2}\left(b_{0}^{2}+F\right)$. This exemplifies our general result that, for any $\mathcal{L}(F)$-theory different from the standard Maxwell vacuum theory, the causality condition is violated for background fields with sufficiently big $G$. 


\subsection{Heisenberg-Euler theory}

The Heisenberg-Euler theory 7 incorporates some effects from quantum electrodynamics into an effective classical theory. We will discuss the approximated Lagrangian up two second order following the notation of Dunne [16]

$$
\mathcal{L}=E_{0}^{2}\left\{-\frac{1}{2} \frac{F}{E_{0}^{2}}+\Lambda\left(\frac{F^{2}}{E_{0}^{4}}+7 \frac{G^{2}}{E_{0}^{4}}\right)\right\}
$$

where

$$
\begin{gathered}
\Lambda=\frac{\hbar c}{90 \pi e^{2}}=0.7363 \\
E_{0}=\frac{m^{2} c^{4}}{e^{3}}=6.048 \times 10^{15} \frac{\sqrt{\mathrm{g}}}{\sqrt{\mathrm{cm}} \mathrm{s}} .
\end{gathered}
$$

The second-order approximation is justified only if the higher-order terms are small, so the allowed field values are restricted by

$$
F^{2}+4 G^{2} \ll E_{0}^{4} .
$$

From the Lagrangian we find

$$
\begin{gathered}
\mathcal{L}_{F}=-\frac{1}{2}+\frac{2 \Lambda F}{E_{0}^{2}}, \quad \mathcal{L}_{G}=\frac{14 \Lambda G}{E_{0}^{2}} \\
\mathcal{L}_{F F}=\frac{2 \Lambda}{E_{0}^{2}}>0, \quad \mathcal{L}_{G G}=\frac{14 \Lambda}{E_{0}^{2}}>0, \quad \mathcal{L}_{F G}=0 .
\end{gathered}
$$

So the first two causality conditions (62) and (63) are obviously satisfied and the third one (64) requires

$$
E_{0}^{2} / \Lambda>F+11 \sqrt{F^{2}+4 G^{2}} .
$$

This is, indeed, satisfied for all allowed field values (100).

\subsection{A pathological example}

For the sake of argument, we consider the Lagrangian

$$
\mathcal{L}(F, G)=b_{0}^{2} \sqrt{1-\frac{F}{b_{0}^{2}}-\frac{G^{2}}{b_{0}^{4}}}-b_{0}^{2}
$$

which results from the Born-Infeld Lagrangian by replacing $(F, G)$ with $(-F, G)$. In a $(3+1)$ splitting this corresponds to interchanging the electric and the magnetic field strength. In comparison to the Born-Infeld case we have also changed the overall sign of the Lagrangian to have again $\mathcal{L}_{F}<0$. Just as the Born-Infeld Lagrangian, the Lagrangian (103) approaches the Maxwell Lagrangian, $\mathcal{L}(F, G) \rightarrow-F / 2$, for $b_{0} \rightarrow \infty$. 
From the Lagrangian we find

$$
\begin{gathered}
\mathcal{L}_{F}=\frac{-1}{2 \sqrt{1-\frac{F}{b_{0}^{2}}-\frac{G^{2}}{b_{0}^{4}}}}, \\
\mathcal{L}_{G}=\frac{-G}{b_{0}^{2} \sqrt{1-\frac{F}{b_{0}^{2}}-\frac{G^{2}}{b_{0}^{4}}}},
\end{gathered}
$$

so we have indeed $\mathcal{L}_{F}<0$ and the constitutive law (5) reads

$$
H^{a b}=\frac{F^{a b}-\frac{G}{b_{0}^{2}} \tilde{F}^{a b}}{\sqrt{1-\frac{F}{b_{0}^{2}}-\frac{G^{2}}{b_{0}^{4}}}} .
$$

The allowed values of $F$ and $G$ are restricted by

$$
1-\frac{F}{b_{0}^{2}}-\frac{G^{2}}{b_{0}^{4}}>0
$$

The second derivative of the Lagrangian with respect to $F$ is given by

$$
\mathcal{L}_{F F}=\frac{-1}{4 b_{0}\left(1-\frac{F}{b_{0}^{2}}-\frac{G^{2}}{b_{0}^{4}}\right)^{3 / 2}},
$$

which implies that $\mathcal{L}_{F F}<0$ for all allowed field values. However, according to (63) $\mathcal{L}_{F F} \geq 0$ is a necessary condition for causality. Therefore, in this case the causality condition is violated for all background fields.

\subsection{Hamiltonian for a regular black hole}

Several regular black holes have been found which are solutions to Einstein's field equation coupled to a nonlinear electrodynamical theory of the Plebański class. Here we consider an example that was found by Ayon-Beato and García 17. For a discussion of general features of such regular black-hole solutions we refer to Bronnikov [18.

The electrodynamical theory is given in terms of a Hamiltonian

$$
\mathcal{H}(R)=\frac{R}{2 \cosh ^{2}\left(\sqrt[4]{R / R_{0}}\right)}
$$

where $R_{0}>0$ is a constant.

The derivatives of the Hamiltonian with respect to $R$ are

$$
\mathcal{H}_{R}=\frac{2-\sqrt[4]{R / R_{0}} \tanh \left(\sqrt[4]{R / R_{0}}\right)}{4 \cosh ^{2}\left(\sqrt[4]{R / R_{0}}\right)}
$$


$\mathcal{H}_{R R}=\frac{\left.-4 \sqrt{R / R_{0}}-5 \sqrt[4]{R / R_{0}} \sinh \left(2 \sqrt[4]{R / R_{0}}\right)+2 \sqrt{R / R_{0}} \cosh \left(2 \sqrt[4]{R / R_{0}}\right)\right)}{32 R \cosh \left(\sqrt[4]{R / R_{0}}\right)}$.

We see that the allowed values of $R$ are restricted by

$$
R \geq 0
$$

because for $R<0$ the Hamiltonian is non-real. As in any other $\mathcal{H}(R)$-theory there is no restriction on the values of $S$. From (110) we read that $\mathcal{H}_{R} \rightarrow 1 / 2$ for $R \rightarrow 0$, so the theory approaches the Maxwell vacuum theory in the weakfield limit as was emphasised in [17.

It is a characteristic feature of this type of regular black-hole solutions that the condition $\mathcal{H}_{R} \neq 0$ does not hold on the entire domain of allowed values, i.e., that $\mathcal{H}$ goes through an extremum at some value $R=R_{m}$. In the example at hand, one can calculate from (110) that $\mathcal{H}$ goes through a maximum at $R_{m} \approx 18.2 R_{0}$. One can perform the passage to the Lagrangian formalism if one restricts either to values $R>R_{m}$ or to values $R<R_{m}$. However, on any interval that contains the point $R=R_{m}$ the relation between $R$ and $F$ is not one-to-one and the passage to the Lagrangian is not possible, cf. Bronnikov [18.

For our investigation of causality we restrict to the interval $0 \leq R<R_{m}$ where $\mathcal{H}_{R}>0$ and we check if the causality conditions (79) and (80) hold on this interval. The first condition is indeed true on the entire interval under consideration. However, the second condition is violated, even for fields with $S=0$ if $R \gtrsim 0.62 R_{0}$. So here we have an example where we do not need a big value of $S$ to violate the causality condition.

\section{Conclusions}

If nonlinear vacuum electrodynamics is realised in Nature, we have to distinguish three light-cones: The light-cone of the space-time metric and the light-cones of the two optical metrics. Light rays are lightlike geodesics of the optical metrics whereas, according to the standard interpretation of (special or general) relativity, the light-cone of the space-time metric determines the maximal speed of signals. If this interpretation is accepted, the optical metrics should be causal in the sense that their lightlike geodesics are lightlike or timelike with respect to the space-time metric. It was the purpose of this paper to characterise those nonlinear vacuum electrodynamical theories for which this is true.

We proceeded in two steps. In the first step, in sections 3 , 4 and 5 , we gave criteria that can be easily checked if the Lagrangian and the background field are specified. These results are complementary to the work of Goulart and Perez Bergliaffa 12 and Abalos et al. 13. In particular, we refer to the latter paper for pictures of how the three light-cones are related in different situations. However, we have prefered to derive our results in the easiest way 
directly from the characteristic equation, without refering to results from the quoted papers. In the second step, in sections 6 and 7 we worked out conditions on the Lagrangian, or on the Hamiltonian, of the electrodynamical theory that guarantee causality of the optical metrics for all allowed background fields. These conditions were given as a set of inequalities the derivatives of the Lagrangian, or of the Hamiltonian, have to satisfy. For an arbitrary $\mathcal{L}(F, G)$ theory, or for an arbitrary $\mathcal{H}(R, S)$-theory, it might be desirable to further investigate these conditions and to characterise in an even more convenient way all theories that guarantee causality. However, for $\mathcal{L}(F)$-theories or $\mathcal{H}(R)$ theories the criteria can be easily evaluated. In particular, we have seen that any such theory other than the standard Maxwell vacuum theory necessarily violates the causality conditions for some allowed background fields.

We have assumed throughout that the underlying space-time metric is the Minkowski metric. However, this is no restriction of generality. As the causality conditions are algebraic conditions on the light-cones, they can be applied on a curved space-time manifold to the light-cones on each tangent space.

We have said that the optical metrics have to be causal if one accepts the hypothesis that the light-cone of the space-time metric determines the maximal speed of signals. We should add that this hypothesis is not totally beyond any doubt. Firstly, one might argue that (tiny) violations might be possible if quantum effects are taken into account, in particular on a curved background, see Drummond and Hathrell 19. However, even in this case our causality conditions remain valid in the sense that they should be satisfied to a very good approximation. Secondly, one might argue that the light-cone of the space-time metric does not determine the maximal speed of signals if there are strong background fields. This is certainly a possibility, but it is tantamount to making major changes to the interpretation of the space-time metric, i.e., to the theory of relativity. At present, we see no compelling reason why one should do this. Therefore, we believe that there are good reasons to consider only those nonlinear vacuum electrodynamical theories as physical which give causal optical metrics for all allowed background fields.

\section{Acknowledgments}

GS wishes to thank Evangelisches Studienwerk Villigst for supporting him with a PhD Stipend during the course of this work. VP is grateful to Deutsche Forschungsgemeinschaft for financial support under Grant No. LA 905/14-1. Moreover, we gratefully acknowledge support from the Deutsche Forschungsgemeinschaft within the Research Training Group 1620 "Models of Gravity". GS also wishes to thank H. v. Borzeszkowski for helpful discussions and comments.

\section{References}

1. G. Mie, Ann. Phys. (Berlin) 37, 511 (1912) 
2. G. Mie, Ann. Phys. (Berlin) 39, 1 (1912)

3. A. Sommerfeld, Electrodynamics: Lectures on theoretical physics, Vol. III (Academic Press, New York, 1952)

4. M. Born, Nature 132, 282 (1933)

5. M. Born, L. Infeld, Proc. Roy. Soc. London A 144, 425 (1934)

6. A.A. Tseytlin, in The many faces of the superworld: Yuri Golfand memorial volume, ed. by M. Shifman (World Scientific, Singapore, 1999)

7. W. Heisenberg, H. Euler, Zeitschr. Phys. 99, 230401 (1936)

8. J. Plebański, Lectures on non-linear electrodynamics (Nordita, Copenhagen, 1968)

9. G. Boillat, J. Math. Phys. 11, 941 (1970)

10. M. Novello, V.A. De Lorenci, J.M. Salim, R. Klippert, Phys. Rev. D 61, 045001 (2000)

11. Y. Obukhov, G. Rubilar, Phys. Rev. D 66, 024042 (2002)

12. E. Goulart, S. Perez Bergliaffa, Class. Quantum Grav. 26, 135015 (2009)

13. F. Abalos, F. Carrasco, E. Goulart, O. Reula, Phys. Rev. D 92, 084024 (2015)

14. G.O. Schellstede, V. Perlick, C. Lämmerzahl, Phys. Rev. D 92, 025039 (2015)

15. R. Courant, D. Hilbert, Methods of mathematical physics, Volume II (Wiley Interscience Publishers, New York, 1962)

16. G. Dunne, Int. J. Mod. Phys. A 27, 1260004 (2012)

17. E. Ayon-Beato, A. García, Phys. Lett. B 464, 25 (1999)

18. K.A. Bronnikov, Phys. Rev. D 63, 044005 (2001)

19. I.T. Drummond, S.J. Hathrell, Phys. Rev. D 22, 343 (1980) 